\title{
Les cahiers de sciences humaines et l'anthropologie du développement (1980-90)
}

Jean Schmitz

\section{OpenEdition}

1 Journals

Édition électronique

URL : http://journals.openedition.org/apad/372

DOI : 10.4000/apad.372

ISSN : 1950-6929

Éditeur

LIT Verlag

Édition imprimée

Date de publication : 15 décembre 1991

\section{Référence électronique}

Jean Schmitz, «Les cahiers de sciences humaines et l'anthropologie du développement (1980-90)», Bulletin de l'APAD [En ligne], 2 | 1991, mis en ligne le 30 juin 2006, consulté le 15 septembre 2020. URL : http://journals.openedition.org/apad/372

Ce document a été généré automatiquement le 15 septembre 2020.

Bulletin de I'APAD 


\title{
Les cahiers de sciences humaines et l'anthropologie du développement (1980-90)
}

\author{
Jean Schmitz
}

1 La pertinence économique du développement a été remise en cause avec la sécheresse qui a sévi dans le monde tropical durant les années 1970, la chute des cours des matières premières, le problème de la dette, etc. Depuis peu, par un retournement paradoxal, la lutte contre le sous-développement est même rendue responsable de la dégradation de l'environnement : déforestation, érosion des sols, désertification, etc... D'où le souci de proposer un développement "durable", susceptible de s'auto-entretenir à long terme. Les opérations de développement "intégrées", comportant de multiples volets et précédées par des recherches interdisciplinaires se sont alors multipliées. A partir des années 1975, l'ouverture, en particulier en direction des sciences sociales, est devenue une "obligation". La revue Les cahiers O.R.S.T.O.M., Série Sciences Humaines, devenue entre temps Les Cahiers des Sciences Humaines, a consacré un certain nombre de numéros spéciaux aux différentes modalités de l"'exercice" du développement en Afrique, Amérique Latine et Asie, dont nous présentons ici quelques exemples significatifs.

2 L'arbre en Afrique tropicale. La fonction et le signe : XVII, 3-4, 1980 (320 p.). Selon Paul Pélissier, auteur de la préface, l'approche proposée par les différentes contributions de ce Cahier est de déchiffrer les termes de l'alliance entre les civilisations africaines et le couvert végétal qui leur sert de cadre, de regarder l'arbre comme l'élément primordial de l'environnement et témoignant de la société dont ce dernier est largement le produit. La jachère forestière est le principal agent de protection et d'enrichissement du sol. Les peuplements ligneux fournissent également toute une gamme de produits de cueillette. De plus, la dernière sécheresse a mis en évidence l'importance du pâturage aérien dans le domaine pastoral. Les fonctions agronomiques, alimentaires, thérapeutiques et zootechniques de l'arbre ne doivent pas faire oublier que celui-ci est également un signe : marqueur d'organisations sociales 
égalitaires utilisant des barrières végétales pour se protéger, emblème identitaire dans le cas des "bois sacrés" ; enfin, signe foncier, trace d'occupation et de mise en valeur.

3 Médecines et santé : XVIII, 1-2, 1981-82 (189 p.). La santé, et plus précisément la maladie et ses représentations, constituent depuis quelques années un des champs de recherche privilégié de l'anthropologie sociale. L'expression "anthropologie de la maladie", sur laquelle la plupart s'accordent pour désigner ce domaine de recherche, recouvre une multiplicité de thèmes : classification des maladies, pratiques et savoirs concernant le corps, élaboration des remèdes et en particulier utilisation des plantes, causalités de la maladie. Tous ces éléments constitutifs de savoirs thérapeutiques prennent sens et trouvent leur cohérence dans la mesure où ils peuvent être rapportés à un système de représentation propre aux sociétés africaines, océaniennes et amérindiennes évoquées.

4 Les instruments aratoires en Afrique tropicale. La fonction et le signe : XX, 3-4, 1984 (658 p.). Après la dernière synthèse sur le sujet, qui date des années 60, ce Cahier représente une nouvelle tentative de recueil de matériaux et de réflexions consacrés à la technologie agricole en milieu tropical. Les contributions rassemblées ici ne se limitent pas à la description morphologique des "objets", renvoyant à une perspective muséographique, mais essaient de se situer au croisement des deux axes de l'étude technique des instruments agricoles : celui des itinéraires techniques et celui des choix opératoires. D'où des tentatives de définition d'invariants, de profil technologique, sans oublier l'étude de la gestuelle, liée aux techniques du corps, à la charnière du fait technique et de la réalité culturelle.

5 Anthropologie et histoire, 21, 1, 1985 (170 p.). Les différentes méthodes de travail des anthropologues étudiant l'histoire des sociétés en Afrique, Amérique et Océanie sont exposées ici. Pour l'Afrique, il s'en dégage que les principales orientations consistent à remettre en cause l'appréciation jusqu'alors portée sur l'entreprise coloniale, à reconsidérer le rapport du "contemporain" au "traditionnel", à s'interroger sur la réalité de l'ethnie, de l'autochtonie, de l'État, etc. Pour l'Amérique et l'Océanie, la réflexion sur l'histoire se centre plutôt autour de thèmes tels que l'identité et la culture. Marqués par une diversité qui n'exclut pas la convergence, ces textes font pleinement apparaître toute l'importance du questionnement que suscite aujourd'hui la relation à l'histoire des sociétés longtemps dites "traditionnelles".

6 Frontières : mythes et pratiques (Brésil, Nicaragua, Malaysia), 23, 3-4, 1987 (259 p.). Avant d'être un ensemble de phénomènes concrets et divers d'occupation de zones pionnières, la frontière est une construction idéologique, un mythe fondateur pour les sociétés dans lesquelles l'expansion territoriale est synonyme d'accession à la richesse. Investie à tous les niveaux par l'État, la frontière symbolise les relations que les hommes entretiennent avec leur territoire. Les études de cas et les analyses théoriques de ce Cahier, qui concerne surtout le Brésil, s'interrogent sur la signification et la portée du processus, sur l'accélération du cycle peuplement/expulsion de ces espaces réputés vides : espaces de la forêt amazonienne, des terres de cerrados ou d'anciennes zones de frontières qu'un autre type de mise en valeur a réactivés; vides démographique, économique ou juridique.

7 Systèmes de production agricole en Afrique tropicale., 23, 3-4, 1987 (259 p.) et 24, 1, 1988 (166 p.), réédité en 1990. Cet ensemble de 21 textes réunit les points de vue de chercheurs en sciences sociales mais aussi d'agronomes et de zootechniciens. Ces contributions constituent une somme très structurée d'études de cas sur les milieux 
ruraux de l'Afrique de l'Ouest francophone et de Madagascar. Après une substantielle partie théorique, les systèmes de production agricole sont examinés dans leur rapport avec le groupe domestique puis avec les interventions de développement et, enfin, dans une perspective dynamique saisissant la pleine dimension des changements en cours.

8 La pêche : enjeux de développement et objets de recherche, 25, 1-2, 1989 (294 p.). Lorsqu'elle n'est pas régulée, l'exploitation d'une ressource vivante renouvelable et "non appropriée" tend vers de telles baisses de rendement que sa rentabilité économique n'est plus assurée. C'est sur ce paradigme que repose la délimitation du champ de recherche halieutique. Ce numéro thématique dont les auteurs sont anthropologues, économistes ou géographes aborde des thèmes divers parmi lesquels on peut citer: évaluation ou critique des modèles bio-économiques de gestion des stocks, formalisation des systèmes de rémunération du capital et du travail dans la pêche artisanale, description de la distribution du poisson frais, mais aussi analyse historique des systèmes de gestion et d'appropriation dits "traditionnels", comme de la pêche industrielle.

9 Sociétés pastorales et développement : histoire des politiques, critiques des doctrines, 26, 1-2, 1990. L'objectif de ce Cahier est d'analyser dans une perspective historique et critique le jeu des forces et des influences qui conduit à la mise en place des politiques appliquées au monde pastoral. Cette question est étudiée sous différents angles : critique conceptuelle et histoire des politiques dans leur formulation la plus technique, hydraulique pastorale. Médecine vétérinaire, etc. Toutes ces analyses sont menées sur la base d'études de cas concrets choisis en Afrique sahélienne ou saharienne et au Moyen Orient arabe. 